\title{
Evaluación de la gestión administrativa para mejorar el desempeño laboral en la gestión municipal
}

\author{
Jhon Bautista Fasabi \\ Jhonbautista007@gmail.com \\ Escuela de posgrado \\ Universidad César Vallejo \\ ORCID: 0000-0003-0809-8463 \\ José Manuel Delgado Bardales \\ jmdelgadob@ucvvirtual.edu.pe \\ Escuela de posgrado \\ Universidad César Vallejo \\ ORCID:0000-0001-6574-2759 \\ Scopus autor ID: 24070333700 \\ Código Renacyt: P0050554
}

\section{RESUMEN}

El objetivo de la investigación fue la de determinar la gestión administrativa para mejorar el desempeño laboral en una gestión municipal, la investigación fue de tipo aplicada, la unidad de análisis está comprendida por los trabajadores administrativos de la Municipalidad Provincial de San Martin. La técnica utilizada fue la encuesta para la recolección de información, se ha evidenciado que algunas municipalidades no llevan a cabo una gestión eficiente, debido a que, anualmente estas devuelven un porcentaje considerable del presupuesto asignado para realizar los proyectos planeados. Se tuvo entrevistas informales con el personal administrativo, el cual ha puesto en evidencia que no se ha gestionado una planificación de actividades durante el COVID, lo cual, no les ha facilitado el desarrollo de estrategias adecuadas para atender las necesidades de los pobladores, además, la comunicación entre las áreas es muy pobre lo que ha dificultado aún más mantener un control sobre las actividades que se lograron organizar, además de los hechos mencionados hasta el momento se ha notado que algunos de los trabajadores no tienen en claro cuáles son sus responsabilidades según el cargo que poseen.

Palabras clave: Gestión; desempeño; laboral. 


\title{
Evaluation of administrative management to improve job performance in municipal management
}

\begin{abstract}
The objective of the research was to decide administrative management to improve job performance in a municipal management, the research was of an applied type, the unit of analysis is comprised of the administrative workers of the Provincial Municipality of San Martin. The technique used was the survey for the collection of information, it has been shown that some municipalities do not carry out an efficient management, due to the fact that annually they return a considerable percentage of the budget assigned to carry out the planned projects. Informal interviews were held with the administrative staff, which has shown that a planning of activities has not been managed during the COVID, which has not facilitated the development of adequate strategies to meet the needs of the residents, in addition, communication between the areas is very poor, which has made it even more difficult to maintain control over the activities that were organized, in addition to the events mentioned so far, it has been noted that some of the workers are not clear about their responsibilities according to the position they hold.
\end{abstract}

Keywords: Management; performance; labor.

Artículo recibido: 03 nov. 2020

Aceptado para publicación: 07 dic. 2020 Correspondencia jhonbautista007@gmail.com

Conflictos de Interés: Ninguna que declarar 


\section{INTRODUCCIÓN}

A nivel mundial se ha generado un estado de emergencia producto de la pandemia del COVID-19, lo cual ha generado preocupaciones a nivel social y económico, esto desde la perspectiva municipal ha generado un gran desafío, puesto que, su eficiencia es crucial para mantener el bienestar de los ciudadanos mediante el gasto público para atender diversas problemáticas que se han presentado en las localidades como una secuela producida por la pandemia (Kulkarni \& Anantharama, 2020, p.1).

Por otra parte, Silva et al. (2018), evidencia que el desempeño laboral como una parte de la gestión administrativa es esencial, puesto que, los profesionales que a nivel municipal integran dicha gestión tienen el propósito de cuidar los bienes estatales y deben manejar de forma adecuada los fondos brindados para otorgar un servicio adecuado a los ciudadanos que se ven beneficiados a través de su desempeño, por ende, conocer el desempeño laboral de los colaboradores municipales es relevante para la organización, ya que, este incide sobre la productividad en este ámbito y sobre cualquiera (p. 57). Por tanto, se refiere que la gestión administrativa y el desempeño laboral se asocian de manera directa, es decir, al mejorar una se verá beneficiada la otra variable.

Además, Kalgin et al. (2018) Manifestaron que la gestión administrativa en las municipales tiene un impacto positivo sobre el desempeño de los colaboradores de primera línea, dado que, sobre la base de la planificación, organización y control se ve influenciado el desempeño, es decir, si esto está establecido de manera adecuada los colaboradores tienen en claro sus actividades y el tiempo requerido para las mismas (pp .65-66). En este sentido, se puede decir que todas las actividades desarrolladas en los escalones superiores penetran en toda la jerarquía y esto se ve repercutido sobre el desempeño de los colaboradores, ya que esto, se desenvuelven sobre la red laboral desarrollada por los líderes.

Asimismo, en el Perú, se ha evidenciado que algunas municipalidades no llevan a cabo una gestión eficiente, debido a que, anualmente estas devuelven un porcentaje considerable del presupuesto asignado para realizar los proyectos planeados anualmente, en base a esta situación en el año 2009 se puso en vigencia la Ley $\mathrm{N}^{\circ}$ 29332, misma que tiene por objetivo mejorar los niveles de recaudación e implementación del gasto en inversiones, desde entonces el Ministerio de Economía y Finanzas (MEF), ha estado desarrollando programas de incentivos para mejorar la gestión administrativas a nivel 
nacional. Por otra parte, se observa que el desempeño laboral en San Martin durante la pandemia del COVID-19 no ha resultado eficiente completamente, dado que, la Defensoría del pueblo ha puesto en evidencia que (2020) algunas actividades no han llevado continuidad o no se activaron los protocolos adecuados en el tiempo esperado, por ejemplo, no se han asegurado la continuidad de las actividades de forma remota en la región, debido a que, la mayoría de las municipalidades presentan una infraestructura inadecuado lo cual ha dificultado la continuidad de las actividades laborales.

Sin embargo, en la Municipalidad Provincial de San Martín se tuvo entrevistas informales con el personal administrativo, el cual ha puesto en evidencia que no se ha gestionado una planificación de actividades durante el COVID, lo cual, no les ha facilitado el desarrollo de estrategias adecuadas para atender las necesidades de los pobladores, además, la comunicación entre las áreas es muy pobre lo que ha dificultado aún más mantener un control sobre las actividades que se lograron organizar, esto ha repercutido sobre la medición de los resultados de su labor. Además de los hechos mencionados hasta el momento se ha notado que algunos de los trabajadores no tienen en claro cuáles son sus responsabilidades según el cargo que poseen, así mismo, se ha evidenciado que les designan una actividad y sin haberla culminado le están solicitando que realice otra, lo cual ha dejado una serie de labores inconclusas que nadie ha logrado finiquitar.

Adicional a ello, se observó que los colaboradores no tienen noción del trabajo en equipo, ya que, sus funciones son realizadas de forma individual cuando deberían ser efectuadas mediante un grupo de trabajo; por otro lado, en cuanto al deseo de participar en el desarrollo de actividades es escaso porque algunos colaboradores no suelen aportar ideas que fomente una mejora en los servicios brindados por la municipalidad. Toda esta situación ha puesto en preocupación a los colaboradores dado que, no tienen en claro que está afectando su trabajo, es por ello que a partir de esto se planteó como problema general de investigación ¿En qué medida la propuesta de gestión administrativa mejora el desempeño laboral en la Municipalidad Provincial de San Martín, 2020? y los objetivos plasmados en el estudio a nivel general se propuso determinar una propuesta de gestión administrativa para mejorar el desempeño laboral en la Municipalidad Provincial de San Martín, 2020. 


\section{ESTRATEGIAS METODOLÓGICAS O MATERIALES Y MÉTODOS}

La investigación fue de tipo básica y el diseño fue no experimental de revisión sistemática, las revisiones sistemáticas son investigaciones científicas en las cuales la unidad de análisis son los estudios originales primarios, constituyen una herramienta esencial para sintetizar la información científica disponible, incrementar la validez de las conclusiones de estudios individuales e identificar áreas de incertidumbre donde sea necesario realizar investigación.

La recolección de datos se realizó a través de la revisión bibliográfica de artículos de investigaciones tanto nacionales como internacionales que tuvieron como tema principal, una gestión administrativa municipal de calidad para la mejora de las condiciones laborales de los trabajadores; de todos los artículos que se encontraron, se incluyeron los más importantes según nivel de evidencia y se excluyeron los menos relevantes. Se estableció la búsqueda siempre y cuando se tuvo acceso al texto completo del artículo científico.

La técnica de análisis fue la revisión sistemática evaluando cada uno de los artículos para una comparación de los puntos o características en las cuales concuerda y los puntos en los que existe discrepancia entre artículos nacionales e internacionales. Además, de acuerdo a criterios técnicos pre establecidos, se realizó una evaluación crítica e intensiva de cada artículo, a partir de ello, se determinó la calidad de la evidencia y la fuerza de recomendación para cada artículo. 


\section{RESULTADOS Y DISCUSIÓN}

RESULTADOS

3.1. Tablas 1: Estudios revisados sobre las prácticas administrativas en el desempeño laboral con referencia a los bancos públicos.

DATOS DE LA PUBLICACIÓN

\begin{tabular}{|c|c|c|c|c|c|c|}
\hline 1. Autor & Año & \multicolumn{3}{|c|}{ Nombre de la Investigación } & \multirow{2}{*}{$\begin{array}{c}\text { Revista donde se ubica la } \\
\text { Publicación } \\
\text { http:// } \\
\text { https://www.semanticschol } \\
\text { ar.org/paper/Impact-of- } \\
\text { Administrative-Practices- } \\
\text { on-Job-With-to-Jayarathna- } \\
\text { Weerakkody/4792e28dfae3 } \\
\text { 0418c032fa0f0ae0e385bcd } \\
\text { 7ac5b } \\
\end{array}$} & $\begin{array}{r}\text { Volumen } \\
\text { y Número }\end{array}$ \\
\hline $\begin{array}{l}\text { S.M.D.Y Jayarathna, W.A.S } \\
\text { Weerakkody }\end{array}$ & I.S 2014 & $\begin{array}{l}\text { Impact Of Admi } \\
\text { Performance W } \\
\text { In Sri Lanka }\end{array}$ & $\begin{array}{l}\text { istrative Practic } \\
\text { h Reference To }\end{array}$ & $\begin{array}{l}\text { Public Banks } \\
\text { Pu Job }\end{array}$ & & $\begin{array}{l}\text { VOLUME } \\
3 \text {, ISSUE 4, }\end{array}$ \\
\hline \multicolumn{7}{|c|}{ CONTENIDO DE LA PUBLICACIÓN } \\
\hline $\begin{array}{l}\text { Tipo y Diseño de } \\
\text { Investigación }\end{array}$ & oblación y Muestra & $\begin{array}{c}\text { Instrumento } \\
\mathrm{s}\end{array}$ & $\begin{array}{l}\text { Aspectos } \\
\text { ético }\end{array}$ & Resultados & Conclusión & \\
\hline $\begin{array}{l}\text { Descriptiva, } \\
\text { relacional, } \\
\text { cuantitativa y no } \\
\text { experimental }\end{array}$ & 92 colaboradores & Cuestionario & $\begin{array}{l}\text { Código de } \\
\text { ética en } \\
\text { investigación }\end{array}$ & $\begin{array}{l}\text { According to the } \\
\text { results, there is } \\
\text { an impact on } \\
\text { administrative } \\
\text { practices on job } \\
\text { performance, but } \\
\text { it is not } \\
\text { significant. }\end{array}$ & $\begin{array}{l}\text { Based on that it can be } \\
\text { s employee's job perform } \\
\text { been impacted by adminis } \\
\text { practices, and the job p } \\
\text { can be improved by deve } \\
\text { t administrative practices. } \\
\text { t }\end{array}$ & $\begin{array}{l}\text { concluded } \\
\text { ance have } \\
\text { trative } \\
\text { erformance } \\
\text { loping the }\end{array}$ \\
\hline
\end{tabular}




\section{Autor}

Año

Hadis Alipoor, Keyvan Ahmadi, Salah Pouya, Khabat Ahmadi, Soran
The Effect of Organizational Structure on Employees' Job Performance in Private Hospitals of Ahvaz
Revista donde se ubica la Publicación

http://www.informaticsjournals.com /index.php/JEOH/article/view/19831 \#: :text=This\%20study\%20aims\%2 0to $\% 20$ determine, a $\% 20$ private $\% 20 \mathrm{~h}$ ospital\%20in\%20Ahvaz.\&text=The $\% 20$ results\%20indicate $\% 20$ that $\% 20$ organizational,a\%20private\%20hosp ital\%20of\%20Ahvaz.

$\begin{gathered}\text { Volumen Y } \\ \text { Numero }\end{gathered}$
Vol 17(3\&4), DOI
$:$
$10.18311 /$ jeoh/2
017/19831, 119-
123, July-
December 2017

\section{CONTENIDO DE LA PUBLICACIÓN}

\begin{tabular}{|c|c|c|c|}
\hline $\begin{array}{l}\text { Tipo y Diseño de } \\
\text { Investigación }\end{array}$ & $\begin{array}{c}\text { Población y } \\
\text { Muestra }\end{array}$ & Instrumentos & $\begin{array}{c}\text { Aspectos } \\
\text { ético }\end{array}$ \\
\hline
\end{tabular}

La población estadística incluye a todos los empleados del hospital Cuantitativo, mencionado en junio descriptivo, transversal relacional y no experimental de 2016 como 589 personas, en el cual el volumen de muestra se calculó como 239 empleados utilizando la fórmula de Cochran

\section{Resultados}

Los resultados indican que la estructura organizacional (aspectos estructurales) tiene un efecto negativo significativo en el desempeño laboral de los empleados en un hospital

Cuestionarios

Consentimien to informado privado de Ahvaz. Es decir, cuanto mayor sea la normativa y complejidad en el nivel (vertical, horizontal, geográfico), formalidad $\mathrm{y}$ concentración organizativa, se reduce el desempeño laboral de los empleados.

\section{Conclusión}

Finalmente, se concluye que se debe intentar disminuir el ritmo de complejidad, formalismo y concentración del hospital y avanzar hacia la estructura orgánica para poder crear motivación y satisfacción laboral en los empleados y animarlos a trabajar, esforzarse y entregar mejores y más servicios calificados a los pacientes, como resultado el trabajo de los empleados. También aumentará el rendimiento y la productividad. 
1. Autor

Año

3. Emad Abed Gan ah reh, Bunyamin Bello, Md. Faruk Abdullah
2018

The impact of administrative control on employees' performance: evidence from industrial companies in Jordan

\begin{tabular}{lc}
$\begin{array}{c}\text { Revista donde se ubica la } \\
\text { Publicación }\end{array}$ & $\begin{array}{c}\text { Volumen Y } \\
\text { Numero }\end{array}$ \\
\hline https://www.researchgate.net/publ & \\
\hline ication/338421436_THE_IMPAC & Volumen: 3 \\
\hline T_OF_ADMINISTRATIVE_CO & números: 10 \\
\hline NTROL_ON_EMPLOYEES'_PE & [Junio, 2018] pp \\
\hline RFORMANCE_EVIDENCE_FR & 5.2 \\
\hline OM_INDUSTRIAL_COMPANIE & \\
\hline S_IN_JORDAN & \\
\hline
\end{tabular}

\section{CONTENIDO DE LA PUBLICACIÓN}

\begin{tabular}{|c|c|c|c|c|c|}
\hline $\begin{array}{l}\text { Tipo y Diseño de } \\
\text { Investigación }\end{array}$ & $\begin{array}{l}\text { Población y } \\
\text { Muestra }\end{array}$ & Instrumentos & Aspectos ético & Resultados & Conclusión \\
\hline $\begin{array}{l}\text { Descriptivo, no } \\
\text { experimental, } \\
\text { cuantitativo }\end{array}$ & $\begin{array}{c}\text { Estuvo } \\
\text { conformada } \\
\text { por un total } \\
\text { de } 433 \\
\text { colaboradore } \\
\text { s de } 63 \\
\text { empresas } \\
\text { industriales }\end{array}$ & Cuestionario & $\begin{array}{l}\text { Código de ética } \\
\text { en investigación }\end{array}$ & $\begin{array}{l}\text { Los principales } \\
\text { resultados demostraron } \\
\text { que el 75\% muestra un } \\
\text { desempeño laboral bajo, } \\
\text { esto puede deberse } \\
\text { atribuido a que el control } \\
\text { administrativo carece de } \\
\text { planificación y y } \\
\text { organización, muchos de } \\
\text { los colaboradores no } \\
\text { tienen en claro sus } \\
\text { actividades ni funciones. }\end{array}$ & $\begin{array}{l}\text { Se concluyó que se evidencia un } \\
\text { impacto del control } \\
\text { administrativo sobre el } \\
\text { desempeño de los colaboradores } \\
\text { con una significancia menor al } \\
5 \% \text { y un coeficiente de } 0,567 \text {, } \\
\text { además se evidencia que la falta } \\
\text { de control administrativo, } \\
\text { dirección y planificación afectan } \\
\text { los deberes y responsabilidades } \\
\text { de los colaboradores, ya que, la } \\
\text { falta de claridad en políticas, } \\
\text { comunicación, distribución de } \\
\text { tareas no les permite culminar } \\
\text { sus actividades. }\end{array}$ \\
\hline
\end{tabular}


DATOS DE LA PUBLICACIÓN

\begin{tabular}{|c|c|c|c|c|}
\hline 4. Autor & Año & $\begin{array}{l}\text { Nombre de la } \\
\text { Investigación }\end{array}$ & $\begin{array}{l}\text { Revista donde se ubica la } \\
\text { Publicación }\end{array}$ & $\begin{array}{c}\text { Volumen Y } \\
\text { Numero }\end{array}$ \\
\hline $\begin{array}{l}\text { Robert M. Verburg, Ann- } \\
\text { Marie Nienaber, Rosalind } \\
\text { H. Searle, Antoinette } \\
\text { Weibel, Deanne N. Den } \\
\text { Hartog, and Deborah E. } \\
\text { Rupp. }\end{array}$ & 2018 & $\begin{array}{l}\text { The Role of Organizational } \\
\text { Control Systems in } \\
\text { Employees' Organization } \\
\text { Trust and Performance } \\
\text { Outcomes }\end{array}$ & $\begin{array}{l}\begin{array}{c}\text { journals.sagepub.com/home/gom } \\
\text { https://doi.org/10.1177/105960111772 }\end{array} \\
\underline{5191}\end{array}$ & Vol. 43(2) 179-206 \\
\hline
\end{tabular}

CONTENIDO DE LA PUBLICACIÓN

\begin{tabular}{|c|c|c|c|c|c|}
\hline $\begin{array}{l}\text { Tipo y Diseño de } \\
\text { Investigación }\end{array}$ & $\begin{array}{c}\text { Población y } \\
\text { Muestra }\end{array}$ & Instrumentos & Aspectos ético & Resultados & Conclusión \\
\hline $\begin{array}{c}\text { Relacional, transversal, } \\
\text { cuantitativa y no } \\
\text { experimental }\end{array}$ & $\begin{array}{l}\text { De los } 206 \\
\text { empleados que } \\
\text { fueron invitados } \\
\text { a participar, } 110 \\
\text { empleados } \\
\text { (junto con sus } \\
\text { supervisores) } \\
\text { participaron en } \\
\text { el estudio, lo } \\
\text { que arrojó una } \\
\text { tasa de respuesta } \\
\text { del } 53,4 \% \text {. }\end{array}$ & Cuestionario & $\begin{array}{l}\text { Consentimient } \\
\text { o informado. }\end{array}$ & $\begin{array}{l}\text { Los principales } \\
\text { resultados } \\
\text { demostraron que el } \\
\text { control } \\
\text { organizacional } \\
\text { presenta un nivel } \\
\text { regular por el } 75 \% \text {, } \\
\text { mientras que el } \\
\text { desempeño laboral } \\
\text { se posición como } \\
\text { regular en un 55\%. }\end{array}$ & $\begin{array}{l}\text { Se concluyó que el control } \\
\text { organización tiene una } \\
\text { influencia en el desempeño } \\
\text { laboral, el coeficiente fue igual a } \\
0,562 \text { y la significancia menor al } \\
5 \% \text {, se demuestra con ello que el } \\
\text { control organización mejora el } \\
\text { desempeño de los colaboradores } \\
\text { se verá favorecido. }\end{array}$ \\
\hline
\end{tabular}




\begin{tabular}{|c|c|c|c|c|c|}
\hline 5. Autor & Año & \multicolumn{2}{|c|}{$\begin{array}{l}\text { Nombre de la } \\
\text { Investigación }\end{array}$} & Revista donde se ubica la Publicación & $\begin{array}{c}\text { Volumen Y } \\
\text { Numero }\end{array}$ \\
\hline $\begin{array}{l}\text { Suguna Sinniah, Rames } \\
\text { Kumar Moona Ha } \\
\text { Mohamed, Vimal } \\
\text { Kadiresan, Prem Kuma } \\
\text { Nadarajan, Adam Arif Le } \\
\text { Aik Keang, Azeyan Awee } \\
\text { Che Siti Lazrina Md Lazim }\end{array}$ & $\begin{array}{l}\text { sh } \\
\text { aji } \\
\text { la } \\
\text { ar } \\
\text { ee } \\
\&\end{array}$ & \multicolumn{2}{|c|}{$\begin{array}{l}\text { An Investigation of the Job } \\
\text { Performance of } \\
\text { Administrative Staff in } \\
\text { Government Hospitals, } \\
\text { Malaysia }\end{array}$} & $\begin{array}{l}\frac{\text { https://www.researchgate.net/publication/327501 }}{\text { 629_An_Investigation_of_the_Job_Performance }} \\
\text { of_Administrative_Staff_in_Government_Hosp } \\
\text { itals_Malaysia } \\
\text { DOI: } 10.5539 \text { / ijbm.v13n10p38 }\end{array}$ & $\begin{array}{l}\text { Revista Internacional } \\
\text { de Negocios y } \\
\text { Gestión; Vol. 13, } \\
\text { núm. 10; } 2018\end{array}$ \\
\hline \multicolumn{6}{|c|}{ CONTENIDO DE LA PUBLICACIÓN } \\
\hline $\begin{array}{l}\text { Tipo y Diseño de } \\
\text { Investigación }\end{array}$ & $\begin{array}{c}\text { Población y } \\
\text { Muestra }\end{array}$ & Instrumentos & $\begin{array}{l}\text { Aspectos } \\
\text { ético }\end{array}$ & \multicolumn{2}{|c|}{ Conclusión } \\
\hline \multirow[t]{2}{*}{$\begin{array}{l}\text { Descriptiva, } \\
\text { cuantitativa,no } \\
\text { experimental, } \\
\text { transversal }\end{array}$} & $\begin{array}{l}\text { La muestra fue } \\
\text { de } 170 \text { de una } \\
\text { población de } \\
300 \\
\text { Participantes }\end{array}$ & Cuestionario & $\begin{array}{l}\text { Consentimi } \\
\text { ento } \\
\text { Informado. }\end{array}$ & \multicolumn{2}{|c|}{ 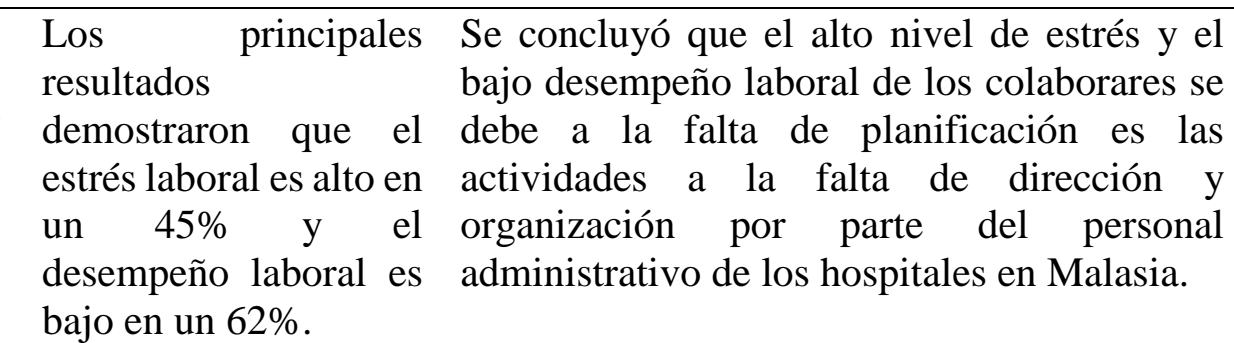 } \\
\hline & Año & \multicolumn{2}{|c|}{ Nombre de la Investigación } & Revista donde se ubica la Publicación & $\begin{array}{c}\text { Volumen Y } \\
\text { Numero }\end{array}$ \\
\hline $\begin{array}{l}\text { Mas Anom bt. Abdu } \\
\text { Rashida, Mohd Noo } \\
\text { Azman bin othmanb, Mohc } \\
\text { Zainudin bin Othmanc } \\
\text { Noor Faizatul Ain b } \\
\text { Arshadd. }\end{array}$ & 2015 & \multicolumn{2}{|c|}{$\begin{array}{lr}\text { THE INFLUENCE OF WORK } \\
\text { ENVIRONMENT } & \text { ON } \\
\text { EMPLOYEES } & \text { JOB } \\
\text { PERFORMANCE: A } & \text { CASE } \\
\text { STUDY } & \text { OF } \\
\text { ADMINISTRATIVE } & \text { STAFF } \\
\text { IN A MANUFACTURING } \\
\text { INDUSTRY }\end{array}$} & $\begin{array}{l}\text { https://www.researchgate.net/publication/29183 } \\
\text { 1102_THE_INFLUENCE_OF_WORK_ENVI } \\
\text { RONMENT_ON_EMPLOYEES_JOB_PERFO } \\
\text { RMANCE_A_CASE_STUDY_OF_ADMINIS } \\
\text { TRATIVE_STAFF_IN_A_MANUFACTURIN } \\
\text { G_INDUSTRY_M }\end{array}$ & $\begin{array}{l}\text { Conference on } \\
\text { Business } \\
\text { Management } \\
\text { Research II (CBMR } \\
\text { II 2015) }\end{array}$ \\
\hline
\end{tabular}




\section{CONTENIDO DE LA PUBLICACIÓN}

\begin{tabular}{|c|c|c|c|c|c|}
\hline $\begin{array}{c}\text { Tipo y Diseño } \\
\text { de Investigación }\end{array}$ & $\begin{array}{c}\text { Población y } \\
\text { Muestra }\end{array}$ & Instrumentos & $\begin{array}{c}\text { Aspectos } \\
\text { ético }\end{array}$ & Resultados & Conclusión \\
\hline $\begin{array}{l}\text { Descriptiva, no } \\
\text { experimental y } \\
\text { cuantitativa }\end{array}$ & $\begin{array}{c}\text { Este estudio } \\
\text { se realizó en } \\
45 \\
\text { encuestados } \\
\text { de la } \\
\text { división de } \\
\text { administraci } \\
\text { ón en el } \\
\text { empresa. }\end{array}$ & Cuestionario & & $\begin{array}{l}\text { El hallazgo muestra } \\
\text { que el entorno laboral } \\
\text { influye en el } \\
\text { desempeño laboral } \\
\text { general, } \\
\text { y entre las tres } \\
\text { dimensiones } \\
\text { estudiadas, el estilo de } \\
\text { comunicación de } \\
\text { liderazgo tiene las } \\
\text { mayores influencias en } \\
\text { el desempeño laboral } \\
\text { de los empleados. }\end{array}$ & $\begin{array}{l}\text { Como conclusión, se evidencia que existe una } \\
\text { relación entre el ambiente laboral como el físico } \\
\text { entorno, estilo de comunicación de liderazgo y } \\
\text { cultura de la organización para el desempeño } \\
\text { laboral de los empleados, sin embargo, fue sólo } \\
\text { a un nivel moderado. Esto significa que hay } \\
\text { evidencia de que el entorno de trabajo influye los } \\
\text { trabajadores sean productivos aparte de otros } \\
\text { predictores. } \\
\text { También es evidencia de que entre los tres (3) } \\
\text { constructos del ambiente de trabajo, la cultura } \\
\text { organizacional es el mayor predictor del entorno } \\
\text { laboral en el desempeño laboral seguido de la } \\
\text { comunicación del liderazgo estilo y por último } \\
\text { ambiente físico. }\end{array}$ \\
\hline
\end{tabular}

5. Autor Año Nombre de la Investigación

Ynzunza, C. Izar, J. Ávila, $\mathrm{R}$

CULTURA ORGANIZACIONAL,
GESTIÓN DE CONOCIMIENTO Y
DESEMPEÑO LABORAL

CULTURA ORGANIZACIONAL, DESEMPEÑO LABORAL

Revista donde se ubica la
Publicación

Volumen Y Numero

Vol. 19,

ISSN: $1405-4574$

laboral 
CONTENIDO DE LA PUBLICACIÓN

\begin{tabular}{|c|c|c|c|c|c|}
\hline $\begin{array}{l}\text { Tipo y Diseño de } \\
\text { Investigación }\end{array}$ & $\begin{array}{l}\text { Población y } \\
\text { Muestra }\end{array}$ & Instrumentos & $\begin{array}{c}\text { Aspectos } \\
\text { ético }\end{array}$ & Resultados & Conclusión \\
\hline Analítico, descriptivo & $\begin{array}{l}\text { El tamaño de la } \\
\text { muestra fue de } \\
247, \text { la escala } \\
\text { utilizada fue tipo } \\
\text { Likert de } 5 \\
\text { puntos para } \\
\text { medir la } \\
\text { gestión de } \\
\text { conocimiento, la } \\
\text { satisfacción y el } \\
\text { desempeño; } \\
\text { mientras que la } \\
\text { cultura } \\
\text { organizacional } \\
\text { se midió con una } \\
\text { escala de 1 a } \\
\text { 100. El total de } \\
\text { ítems fue de } 58 .\end{array}$ & Cuestionario & & $\begin{array}{l}\text { La cultura organizacional } \\
\text { ligeramente predominante es } \\
\text { la de mercado según la escala } \\
\text { de OCAI. Las prácticas de } \\
\text { gestión de conocimiento (GC) } \\
\text { tienen un efecto significativo } \\
\text { sobre los niveles de } \\
\text { satisfacción y el desempeño } \\
\text { laboral; y están vinculadas } \\
\text { positivamente con el uso de } \\
\text { las Tecnologías de la } \\
\text { Información y Comunicación. } \\
\text { Existen diferencias en cuanto al } \\
\text { impacto de la cultura } \\
\text { organizacional sobre la gestión } \\
\text { del conocimiento, al igual que } \\
\text { en los niveles de satisfacción y } \\
\text { el desempeño laboral, } \\
\text { favoreciendo las de aplicación y } \\
\text { conservación del conocimiento } \\
\text { en la mayoría de ellas. } \\
\text { Asimismo, se encontraron } \\
\text { similitudes entre la cultura de } \\
\text { adhocracia y la jerarquizada }\end{array}$ & $\begin{array}{l}\text { La cultura organizacional está } \\
\text { relacionada con la gestión de } \\
\text { conocimiento, fomenta actitudes } \\
\text { positivas hacia éste, generando } \\
\text { niveles más altos de satisfacción; } \\
\text { es un catalizador importante para } \\
\text { el aprendizaje organizacional, la } \\
\text { efectividad laboral y sin duda } \\
\text { alguna, el desempeño } \\
\text { organizacional. La gestión de } \\
\text { conocimiento debe ser una } \\
\text { actividad planeada y } \\
\text { sistematizada que permita la } \\
\text { identificación, generación y } \\
\text { transferencia del conocimiento. }\end{array}$ \\
\hline
\end{tabular}




\begin{tabular}{|c|c|c|c|c|}
\hline 6. Autor & Año & Nombre de la Investigación & Revista donde se ubica la Publicación & $\begin{array}{l}\text { Volumen } \\
\text { Y Numero }\end{array}$ \\
\hline Urbano s. & 2018 & $\begin{array}{l}\text { Clima organizacional y } \\
\text { desempeño laboral de los trabajadores de } \\
\text { la } \\
\text { Administración Local de Agua Huaraz }\end{array}$ & $\begin{array}{c}\text { DOI: } \\
\text { http://dx.doi.org/10.32911/as.2018.v11.n1.46 } \\
5\end{array}$ & $\begin{array}{c}\text { ISSN-L } \\
2616-9541\end{array}$ \\
\hline
\end{tabular}

\section{CONTENIDO DE LA PUBLICACIÓN}

\begin{tabular}{|c|c|c|c|c|c|}
\hline $\begin{array}{c}\text { Tipo y Diseño de } \\
\text { Investigación }\end{array}$ & $\begin{array}{c}\text { Población y } \\
\text { Muestra }\end{array}$ & Instrumentos & $\begin{array}{c}\text { Aspectos } \\
\text { ético }\end{array}$ & Resultados & Conclusión \\
\hline $\begin{array}{ll}\text { Cuantitativo, } & \text { de } \\
\text { diseño } & \text { no } \\
\text { experimental- } & \\
\text { tran-seccional } & \text { y } \\
\text { carácter } & \\
\text { correlacional- } & \\
\text { causa } & \end{array}$ & $\begin{array}{l}\text { La población } \\
\text { está } \\
\text { contituída } \\
\text { por el total de } \\
\text { trabajadores } \\
\text { de la ALA- } \\
\text { HZ, periodo } \\
2016 \text {. El } \\
\text { método para } \\
\text { la elección } \\
\text { de la muestra } \\
\text { fue no } \\
\text { probabilístic } \\
\text { o- } \\
\text { intencional. }\end{array}$ & Cuestionario & & $\begin{array}{l}\text { Se obtuvo que en la dimensión nivel de } \\
\text { percepción global del ambiente laboral } \\
\text { un 54\% de trabajadores calificaron el } \\
\text { clima organizacional como favorable. } \\
\text { Además, la dimensión nivel de } \\
\text { percepción del ambiente laboral en } \\
\text { relación a la infraestructura y } \\
\text { tecnología, refleja que } 8 \% \text { califica el } \\
\text { ambiente como muy favorable. En } \\
\text { relación al desempeño laboral, } 38 \% \text { lo } \\
\text { califica como excelente. }\end{array}$ & $\begin{array}{l}\text { Se concluyó que un adecuado } \\
\text { clima organizacional mejora } \\
\text { el desempeño laboral en los } \\
\text { trabajadores de la AL } \\
\text { A-HZ. }\end{array}$ \\
\hline
\end{tabular}




\section{Autor}

Año

Nombre de la Investigación

Análisis de la Satisfacción y el Desempeño

Laboral en los Funcionarios de la

Municipalidad de Talcahuano
Volumen

Y Numero

Publicación

vol.17 no.5

4

ISSN 0718-

2449

\section{CONTENIDO DE LA PUBLICACIÓN}

\begin{tabular}{|c|c|c|c|c|c|}
\hline $\begin{array}{l}\text { Tipo y Diseño de } \\
\text { Investigación }\end{array}$ & $\begin{array}{c}\text { Población y } \\
\text { Muestra }\end{array}$ & Instrumentos & $\begin{array}{l}\text { Aspectos } \\
\text { ético }\end{array}$ & Resultados & Conclusión \\
\hline $\begin{array}{lr}\text { estudio } & \text { no } \\
\text { experimental, de diseño } \\
\text { transversal, } \\
\text { descriptivo } \\
\text { correlacional }\end{array}$ & $\begin{array}{l}\text { La información } \\
\text { se obtuvo de } 259 \\
\text { funcionarios de } \\
\text { la municipalidad } \\
\text { a quienes se } \\
\text { aplicó un } \\
\text { cuestionario } \\
\text { estandarizado, } \\
\text { personal, } \\
\text { anónimo y } \\
\text { voluntario }\end{array}$ & Cuestionario & & $\begin{array}{l}\text { La fiabilidad de las escalas varía desde } \\
\text { meritorios a excelentes, con tamaño de } \\
\text { Alfa de Cronbach entre } 0,7 \text { y } 0,9 \text {. Los } \\
\text { valores de desempeño laboral se } \\
\text { distribuyen en } 6 \text { escalas, situando a los } \\
\text { encuestados en una evaluación de } \\
\text { "desempeño alta". Mientras que los } \\
\text { valores de satisfacción laboral se } \\
\text { distribuyen en } 10 \text { escalas, situando a } \\
\text { los encuestados en un nivel de } \\
\text { "laboralmente satisfechos", tanto para } \\
\text { la muestra femenina como masculina. }\end{array}$ & $\begin{array}{l}\text { El estudio encontró correlaciones } \\
\text { estadísticamente significativas } \\
\text { entre ambas variables, se destacan } \\
\text { las correlaciones positivas entre las } \\
\text { escalas de desempeño y } \\
\text { satisfacción con la relación con el } \\
\text { jefe, para el género femenino. Y } \\
\text { las escalas de satisfacción con } \\
\text { desempeño en la productividad, } \\
\text { para el género femenino. }\end{array}$ \\
\hline
\end{tabular}




\section{CONCLUSIÓN O CONSIDERACIONES FINALES}

Culminada el análisis sistemático de los artículos científicos, del total revisado, de los países referenciados. El tipo de investigación es descriptiva con sus diferentes variantes.

La mayor parte de las conclusiones de los autores, coinciden en que una buena gestión de la administración repercute en el desempeño laboral, debe estar centrado en las necesidades del ciudadano y que a la vez se refleje en la satisfacción de la misma.

Sin embargo, Bakotic, D sostiene La gestión administrativa es el área responsable de coordinar los recursos administrativos de la empresa. Para llevar a cabo esta tarea, es necesario organizar las necesidades, los procesos y los recursos con los que dispone cada departamento de la empresa y gestionarlos de la mejor manera. Además, Bakotic, D Determinó que la gestión del desempeño laboral se relaciona significativamente con la calidad de servicio público, lo que indica que sí existe una correlación positiva muy alta entre la gestión administrativa y el desempeño laboral A si mismo se estable que el conocimiento, las habilidades y las actitudes de los funcionarios, nos permite demostrar, que existe una correlación positiva muy alta entre el conocimiento y la calidad de servicio público.

Asimismo, Hernández, H., Barrios, I., \& Martínez, D Concluyen que las percepciones de calidad tienen una fuerte influencia con la satisfacción sobre los servicios públicos domiciliarios y, en menor medida, con la satisfacción general sobre la gestión administrativa, a su vez se revela una relación fuerte entre percepciones de calidad y satisfacción.

\section{LISTA DE REFERENCIAS}

Alipoor, H., Ahmadi, K., Pouya, S., Ahmadi, K., \& Mowlaie, S. (2017). The Effect of Organizational Structure on Employees' Job Performance in Private Hospitals of Ahvaz. Journal of Ecophysiology and Occupational Health, 7(3-4), 119-123. Obtenido http://www.informaticsjournals.com/index.php/JEOH/article/view/19831/16483

Alvim, F., \& Ofenhejm, A. (2016). Motivação, satisfação profissional e evasão no serviço público: o caso da carreira de especialistas em Políticas Públicas e Gestão Governamental. 50(1), 17-39. Recuperado el 2019, de http://www.scielo.br/pdf/rap/v50n1/0034-7612-rap-50-01-00017.pdf 
Alvim, F., \& Ofenhejm, A. (2016). Motivação, satisfação profissional e evasão no serviço público: o caso da carreira de especialistas em Políticas Públicas e Gestão Governamental. 50(1), 17-39. Recuperado el 2019, de http://www.scielo.br/pdf/rap/v50n1/0034-7612-rap-50-01-00017.pdf

Bakotic, D. (2015). Relación entre satisfacción laboral y desempeño organizacional. 118130.

Connell, J., Carlton, J., Grundy, A., Taylor, E., Keetharuth, A. D., Ricketts, T., \& Brazier, J. (2018). The importance of content and face validity in instrument development: lessons learnt from service users when developing the Recovering Quality of Life measure (ReQol). Quality of Life Resarch, 27(7), 1893-1902. Obtenido de https://scihub.tw/10.1007/s11136-018-1847-y

Ganahreh, E., Bello, B., \& Abdullah, F. (2018). The impact of administrative control on employees' performance: evidence from industrial companies in Jordan. International Journal of Accounting, Finance and Business, 3(10), 52-66. Obtenido de https://www.researchgate.net/publication/338421436_THE_IMPACT_OF_ADMIN ISTRATIVE_CONTROL_ON_EMPLOYEES'_PERFORMANCE_EVIDENCE_F ROM_INDUSTRIAL_COMPANIES_IN_JORDAN

Hernández, H., Barrios, I., \& Martínez, D. (2018). Gestión de la calidad: elemento clave para el desarrollo de las organizaciones. Criterio Libre, 16(28), 179-195. Recuperado el 2020, de https://core.ac.uk/download/pdf/229911313.pdf

Martínez, A. (2015). Gestión administrativa y desempeño laboral en los trabajadores administrativos de la unidad de administración del Instituto Nacional de Bienestar Familiar 2015. Tesis de Maestria, Universidad Cesar Vallejo. Obtenido de http://repositorio.ucv.edu.pe/handle/20.500.12692/5953

Rashid, A., Othman, M., \& Othman. M Arshad, F. (2015). The influence of work environment on employees job performance: a case study of administrative staff in a manufacturing industry. Conference on Business Management Research II (CBMR II 2015). 
https://www.researchgate.net/publication/291831102_THE_INFLUENCE_OF_WO RK_ENVIRONMENT_ON_EMPLOYEES_JOB_PERFORMANCE_A_CASE_ST UDY_OF_ADMINISTRATIVE_STAFF_IN_A_MANUFACTURING_INDUSTR Y_M

Weerakkody, E., \& Jayarathna, D. (2014). Impact Of Administrative Practices On Job Performance With Reference To Public Banks In Sri Lanka. Internacional Journal of scientific and technology research, 3(4), 162-169. Obtenido de https://www.researchgate.net/publication/280831569_Impact_Of_Administrative_P ractices_On_Job_Performance_With_Reference_To_Public_Banks_In_Sri_Lanka 